\title{
Molecular Dynamics Simulations of Initial Chemical Reaction Mechanism of Shocked CL- 20 Crystals Containing Nanovoids
}

Fuping Wang, Lang Chen*, Deshen Geng, Jianying Lu, Junying Wu

State Key Laboratory of Explosion Science and Technology, Beijing Institute of Technology, Beijing, 100081, China

Supporting Information

\section{Table of Contents}

S1: (a) Velocities of atoms along the $y$ direction and (b) regional temperatures in the central molecular layer of a void with a radius of $4 \mathbf{n m}$ at different times when the impact velocity is $2 \mathrm{~km} / \mathrm{s}$.

* The type of article: Research paper

Corresponding authors: Lang Chen, Tel \& Fax: +86 1068914711.

E-mail: chenlang@bit.edu.cn 


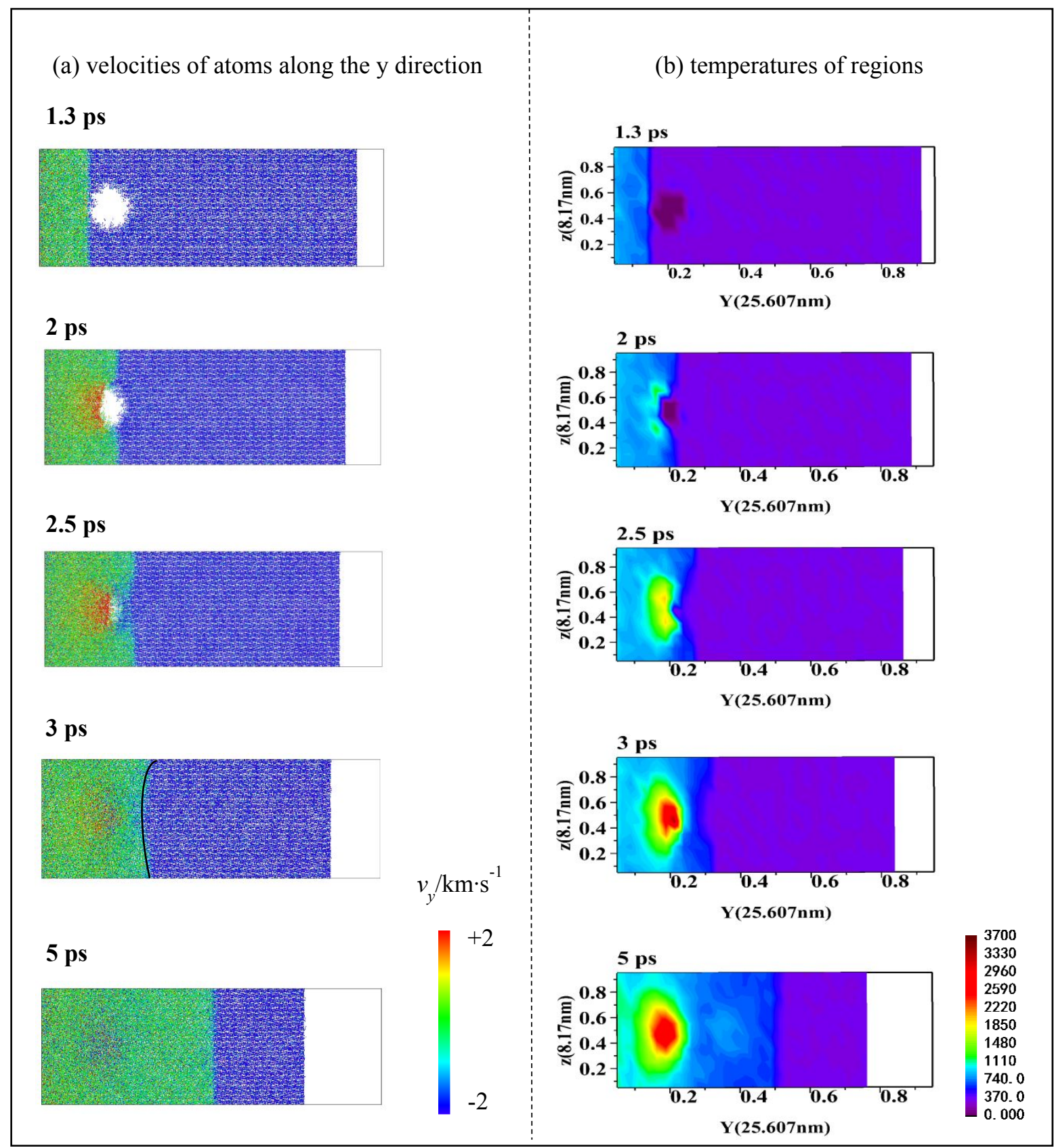

Figure S1 (a) Velocities of atoms along the y direction and (b) regional temperatures in the central molecular layer of a void with a radius of $4 \mathrm{~nm}$ at different times when the impact velocity is $2 \mathrm{~km} / \mathrm{s}$. 